to the purpose than something which appeals to the locality? The doctor, like the application, is "on the spot"; he knows both where the disease is and what will do it good. The blister may irritate the body but it soothes the soul.

Let us act rationally when we can. It is perfectly true that we cannot always be rational, sometimes we must be empirical; we may not know in the least how a thing acts but we may be so certain that it does act that we ask no more; this is the case with colchicum in gout and iodide of potassium in syphilis. But when we propose to ourselves to establish some mode of action let us be sure that we are guided by sound considerations. If we wish to draw blood from an organ by local depletion let us make sure that the vessels upon which we operate have some local connexion with the organ in question. If we propose to relieve by resication let the serum be taken from capillaries which are in communication with those of the suffering part. If we propose to employ counter-irritation let us say to ourselves that irritation is one thing and counter-irritation is another. We know that we can irritate, but as to counterirritation the sceptical may entertain some doubt and those who are content with one affliction may hesitate about the desirability of adding another.

\section{THE DIAGNOSIS OF CERTAIN NOTIFIABLE DISEASES. 1}

BY F. FOORD CAIGER, M.D., M.R.C.P. LOND., D.P.H. Cantab.,

MEDICAL SUPERINTENDENT OF THE SOUTH-WESTERN FEFER HOSPITAL STOCKWELL.

THE accurate and early diagnosis of infective disease always has been, and must necessarily be, a matter of anxious consideration in general practice, if only in view of the importance of early isolation; but in these days of compulsory notification it assumes an exaggerated importance. I shall therefore attempt to the best of my ability to give a brief summary of the relative value of the various symptoms in the diagnosis of some of the infectious fevers with which I have become impressed as the result of 12 years' daily observation. It would obviously be impossible within the limits of a short paper to deal individually with each of the diseases which are notifiable in London; moreover, my object is to introduce what should prove to the members of this society a fertile field for discussion rather than to weary them with a too lengthy dissertation. I propose, therefore, to confine my remarks to the most common, and consequently the most important, members of the group, and for which, moreover, in London the Legislature provides the necessary accommodation-viz., scarlet fever, diphtheria, enteric fever, and small-pox-although the discussion of their differential diagnoses involves a brief allusion to the characteristics of certain other diseases with which they are liable to be confounded.

Scarlet fever.-The points on which the diagnosis of scarlet fever rests are the following. 1. The length of incubation when determinable, which in the large proportion of cases is three days or a little less. It is not often less than 48 hours, and very rarely more than four days, though exceptional cases are on record in which it has been practically proved to have been as long as six days. 2. The fact that the attach is usually ushered in with a combination of sore-throat, headache, and vomiting. Although coupled of course with other general signs of febrile invasion these three symptoms stand out as pre-eminently suggestive of scarlet ferer. The vomiting is often absent in adults, as is the headache, at any rate as an ascertainable fact, in children. The importance of vomiting as an early indication of scarlet fever in children is confirmed by the fact that in a series of 1000 cases in which the history of onset was carefully inquired into vomiting was noted in no less than 80 per cent. of them, and three-fourths of these patients were children. The only other specific fever which shows anything like the same incidence of vomiting is small-pox. 3 . The association of a characteristic puncto-erythematous

1 A paper rearl before the South-West London Medical Society on May 10th, 1899. rash and faucial inflammation with a characteristio appearance of the tongue, dependent on its becoming rapidly denuded of its surface epithelium and so coming to resemble a ripe succulent strawberry. The process of denudation may be often recognised as early as on the second day of fever by the clean red appearance of the tip and edges in contrast with the thick coat of fur which usually covers the rest of the surface. From the edges the stripping process rapidly spreads towards the centre, so that by the third or fourth day of the attack the tongue presents the raw denuded appearance which is so characteristic of scarlet fever. It must be remembered, however, that the completeness with which the tongue peels is proportionate to the degree of inflammatory injection of the faucial and buccal mucous membrane, just as the amount of peeling of the skin is determined by the intensity and persistence of the antecedent eruption. Consequently, in the diagnosis of an attack attended with but slight sore throat we are unable to expect much assistance from the distinctive character of the tongue; and it must be confessed that this is very often the kind of case as to the nature of which there may be considerable doubt. 4. A pulse which at an early stage of the attack is often characterised by undue rapidity. A pulse of from 140 to 160 during the first 36 hours in a child of five years of age should give rise to no anxiety. Towards the end of the second day, even though the temperature may have risen higher, it usually. becomes less rapid and assumes a normal febrile relation. It should be remembered, however, that extreme rapidity of pulse is often an early symptom in pneumonia as well. 5. A temperature the course of which in simple scarlet fever usually conforms to a well-recognised type. The pyrexia, lasting usually from five to seven days, is of a markedly pyramidal shape when charted, with the apex, representing the acme of the fever, either on the third or fourth night. Defervescence takes place gradually by lysis and is usually spread over four days. In view of the misleading statements which are so often made in text-books it cannot be too strongly insisted upon that a true temperature crisis in scarlet fever is excessively rare. In the septic form of attack characterised by destructive ulceration of the fauces the temperature, instead of falling gradually from the third or fourth night, is continued at the same level and assumes a remittent or pyæmic character. In the toxic or so-called "malignant" type of attack, which, happily, in London at the present day is very rarely seen, the temperature remains at a high level, usually between $104^{\circ}$ and $106^{\circ} \mathrm{F}$. until death, which commonly takes place on the fourth or fifth day. 6. The ocourrence of characteristic desquamation. I think the statement is justified that peeling of some degree takes place in all cases which have presented a distinct eruption-at any rate, a punctiform eruption. It may, however, require very careful and minute examination to recognise its presence, particularly in infants and in adults who are endowed with a greasy skin, such as is often seen in young adults the subjects of acne punctata. In an infant so slight and transient may be the peeling that it can only be detected by the nurse in the form of delicate rolls of cuticle which are removed by the towel when the child is being dried after its bath. For this reason the peeling may readily escape observation. To a careful observer the first sign of peeling may be apparent as soon as the second or third day of the eruption in the form of a delicate powdering on the surface of the cheek, which is at that time vividly injected. The result is an appearance of powder and rouge which is strongly suggestive of the gentle art of self-adornment, although it undoubtedly enbances the natural beauty of a beautiful child. In those with a very delicate skin the complexion suggests rather the bloom on a ripe peach. A vividly flushed cheek limited in the same manner by the naso-labial folds is seen in many other acute diseases, notably in acute pneumonia, and is not therefore in itself distinctive, but it is the delicate powdering of the flushed surface which is so eminently suggestive of scarlet fever. Towards the end of the week, before the rash has quite faded, peeling is usually apparent on the neck and on the upper part of the chest, whence it spreads downwards during the course of the ensuing week. On parts of the surface where the skin is normally soft and smooth, as, for instance, the chest, the abdomen, the groins, the inner aspect of the arms, and the thighs, the cuticle separates in the form of delicate shreds or scales. It is more especially in these regions that the desquamation presents certain characteristics which are very distinctive of 
the disease. At the earliest stage as soon as the tension of the skin associated with the inflammatory hyperæmia of the rash has subsided, on careful inspection the most superficial cuticular layer will be found to present a number of small perforations of about the size of a pin's head or larger which give to it a "worm-eaten" appearance, each of the "pinholes" corresponding to one of the finely papular constituents of the recent eruption of which the delicate investing layer has become rubbed off. During the next few days each of these little "pinholes" or rings becomes gradually larger by centrifugal extension until its periphery becomes merged with those which have spread from other and neighbouring centres. As a result anything suggesting this ring-like arrangement then becomes indistinguishable and more or less triangular islets or shreds of cuticle remain, which are ultimately shed, thereby completing the desquamative process so far as that portion of the surface in concerned. The early stage of the peeling, then, is characterised by this "pinhole" appearance of the cuticle and the later stage by these "islets" or irregular shreds of epidermis which are the last to separate. This peculiarity yields trustworthy evidence as to the peeling being scarlatinal ; and, moreover, according to its progress, and the parts of the surface which have become invaded, valuable indications may be derived as to the length of time which has elapsed since the commencement of the attack. 7. Lastly, from the confirmatory evidence which is often afforded by the supervention of one of the well-recognised complications of scarlet fever, chief of which are, of course, otorrhœe, cervical adenitis, nephritis, and rheumatism. There are certain facts connected with the appearance of these complications which are well worth remembering when one is concerned with their value as confirmatory evidence of scarlet fever. The otorrhœa, for instance, is practically limited to young children, its percentage incidence falling progressively from 18.5 in the first quinquennium of life to 0.7 in the fourth quinquennium. The cervical adenitis shows a progressive fall from 11.7 in the first quinquennium to 1.4 in the fourth Renal inflammation, as evidenced by either acute nephritis or simple albuminuria, remains pretty constant at all ages, thcugh definite acute nephritis is relatively rare after 15, years of age. Scarlatinal rheumatism, on the other hand becomes more frequent as age advances, its incidence rising from 0.4 per cent. under five years to 13.6 per cent. in persons aged 20 years and upwards. Another striking point in connexion with the rheumatism is a remarkable constancy in the time of its appearance-viz., almost invariably the fifth, sixth, or seventh day of the attack, at a time when the rash and fever in simple scarlet fever are just disappearing.

These, then, are the main indications on which we rely for the diagnosis of scarlet fever. A well-developed attack should present no difficulty, and there is really no excuse for mistaking it for any other disease. But the case may be very different. It is the ill-developed or negative attacks which so often give rise to very great difficulty and constitute the bete noire of the general practitioner who is but too often expected to be endowed with intuitive perception-the kind of attack which causes one to ask oneself, "Is there sufficient evidence in this case to justify the diagnosis of scarlet fever?" "Must I insist upon a prolonged and often, both to the patient and his friends, an irksome period of isolation which may possibly be, after all, unnecessary?" Or, on the other hand, "Am I justified in letting this patient remain where he is with the ultimate possibility of disaster?" It must be confessed that in some cases the question is unanswerable by any human observer, but $I$ think that if cases be carefully tested by the criteria which I have enumerated their number will not be very considerable.

Now, turning to the differential diagnosis of scarlet fever it may, I think, be fairly stated that there are two classes of ailments with which scarlet fever is most liable to be confounded. First, those in which sore-throat is the prominent feature-viz., simple tonsillitis, diphtheria, and certain cases of influenza; and secondly, those which are attended with a diffused red exanthem-viz., measles, rötheln, and a condition resulting from the administration of certain drugs. Now the differentiation of simple tonsillitis from mild scarlet fever may be an extremely difficult thing, especially in adults, in whom the sore-throat is often accom. panied by a distinct rosy efflorescence on the skin. This, however, is usually limited to the chest and the abdomen, perhaps invading the arms as well; but it is rarely seen on the legs. It lacks the punctate character which is so distinctive of the scarlatinal eruption, being represented by a simple erythematous flush, which, though often vivid at an early stage, rarely persists for more than from 24 to 36 hours and leaves no staining of the skin behind. There is probably an absence of prodromal vomiting and the tongue retains the character which is almost invariably associated with tonsillitis. It is pale and flabby in appearance and is coated with dirty-white pasty fur, so different to the rapidly cleaning tongue of scarlet fever. This distinction is most important as there is no form of sore-throat other than that associated with scarlet fever which is attended with a clean, much less a raw, tongue. Frequent careful and minute inspection of the skin will fail to reveal any indication of peeling, except perhaps on that portion of the neck which has been previously covered with a poultice or a hot fomentation, but even here there is an absence of any "pinhole" arrangement. In an adult or older child the occurrence of rheumatic pains and tenderness in any of the joints, especially in the wrists or the metacarpo-phalangeal joints from five to seven days after the onset of a febrile sore-throat of doubtful nature, is strongly indicative of the case being one of scarlet fever, and the same inference attaches to the appearance of otorrhcea in a young child, though not perhaps appearing until two or three weeks later. Simple albuminuria is as common during the febrile stage of simple tonsillitis as it is in scarlet fever, but the supervention of either simple albuminuria or acute nephritis during the second, third, or fourth week is strongly in favour of the latter. Simple tonsillitis but very rarely occurs in young children. In them a definite inflammation of the faucial structures, except as part of a general catarrh, almost invariably means one of two diseases - viz., scarlet fever or diphtheria.

The differentiation of scarlet fever from diphtheria is often confused owing to the fact not being sufficiently well recognised that an exudation is occasionally present on the tonsils in scarlet fever, which is certainly not diphtheritic. The exudation of scarlet fever, which was, I think, first described by Professor Henoch under the name of "scharlach nekrose," is only found in cases attended with severe faucial inflammation. It is limited to one or both tonsils and does not encroach either on the palate or the uvula. It is more cheesy and friable in consistence and never presents the glistening or fibrous appearance which is so often seen in true diphtheria. Further and more general points in the differentiation between scarlet fever and faucial diphtheria will be referred to when dealing with the latter disease.

The fact that tonsillar or faucial inflammation is occasionally the most obvious manifestation of certain attacks of influenza is responsible for its being sometimes mistaken for scarlet fever, and it is probably within the experience of many practitioners that a whole series of attacks of this protean disease at one time or another have conformed to this type and so contributed to the confusion, at any rate at an early period of the epidemic. The absence of a punctiform eruption and of early cleaning of the tongue, the irregularity of the temperature, the intensity of the prostration, and the relative immunity of young children are points which tend to exclude scarlet fever.

Now, in respect to the eruptive diseases which most fre quently simulate scarlet fever-viz., measles and rötheln (or German measles) - the latter is the one which is most often a source of difficulty, the reason being that both rash and sore-throat are normal accompaniments of rötheln. The differentiation from scarlet fever is sometimes exceedingly difficult. The points on which reliance must be placed are the following: 1. A longer period of incubation, usually 10 days or rather more. 2. An absence of prodromal vomiting and actual pain in swallowing. 3 . The rash is usually the first sign, indeed, it may be the only sign, of illness. 4. The temperature is frequently normal throughout and is rarely raised for more than from 24 to 48 hours. 5. The eruption is usually measly at an early stage, though the spots are usually pinker, smaller, and more discrete than in true measles. At a later stage, usually by the second day, the eruption tends to become erythematous on the trunk, when no suggestion of individual spots can be made out, and this is the time when its likeness to scarlet fever is most confusing. Unlike scarlet fever, however, definite spots are to be seen on the face in most cases, though rarely visible there for more than from 36 to 48 hours. The eruption generally will in all probability have disappeared, eren from the legs, by the end of 
the third day, though a very faint mottling may still be just visible in a good light on the extremities. 6. Nothing, more than slight redness and catarrh will be found in the fauces, and it is not ancommon for the catarrh to affect the naso-lacrymal mucous membrane to a slight extent. 7. The tongue never becomes unduly clean, much less raw, and though slight and transient peeling, perhaps more pronounced than in the average case of measles, may be seen on the trunk and limbs it is never of the "pinhole" variety and stops short of the palms and soles. 8. The most striking distinction which rötheln commonly exhibits, as opposed to scarlet fever, is a tender enlargement of the concatenate chain of glands in the posterior cervical region or of those lying over the mastoid bones. They may be slightly enlarged in scarlet fever, it is true, but never, I believe, tender; whereas in rötheln it is not uncommon for an adult to voluntarily complain of tenderness in this region even for a day or two before the rash comes out. This complaint of tenderness on the part of the patient is most important.

Now, as regards true measles the points of distinction between it and scarlet fever are so well recognised that 1 hesitate to reiterate them in detail. The points, however, which present themselves prominently to one's mind are the following. 1. The comparatively long incubation of measles. 2. The presence of definite lacrymal, nasal, and respiratory catarrh, usually for two or three days before the rash appears giving the patient an aspect of unalloyed misery. 3. The temporary decline of temperature just before the rash comes out and the re-access of pyrexia on its appearance. 4. The spotty character of the eruption as opposed to the minute punctation and confluent flush of that of scarlet fever-the fact that the spots usually are first discernible at the sides of the forehead and behind the ears at the margin of the hairy scalp and that they definitely invade the face, and especially the region round the mouth, which in scarlet fever remains pale and unaffected. 5. The fact that there is no definite inflammation of the faucial structures except as a part of a general catarrhal affection of the mucous surfaces, and certainly no ulceration of the tonsils with enlargement of the subjacent lymphatic glands. 6. The liability to the supervention of bronchial or pulmonary inflammation and the absence alike of those complications and the subsequent desquamation which are so characteristic of scarlet fever. 7. Lastly, the fact that the temperature in uncomplicated attacks falls suddenly by crisis within 36 hours of the appearance of the eruption, instead of declining gradually hand-inhand with it, as is the rule in scarlet fever.

These are the points which usually suffice to make the distinction easy. When we are confronted with a case which gives rise to any difficulty it is usually in respect to a case of scarlet fever which presents characters suggestive of measles, rather than a case of measles simulating the appearance of scarlet fever. Cases of scarlet fever do sometimes occur in which there is an amount of conjunctival injection with obvious lacrymation at an early stage which are not unlike measles, and the rash in parts may be of a blotchy and decidedly morbilliform character which further tends to heighten the similarity. The conjunctival injection, however, passes off after the first 24 hours and does not extend to the adjacent mucous surfaces, while the measly character of the rash is usually confined to the limbs and never invades the face or circum-oral region.

Now, of the drugs which are liable in certain persons to set up an exanthem which may simulate the eruption of scarlet fever the only ones which have fallen within my own experience are copaiba, salicylic acid, antipyrin, belladonna, and iodoform. The rashes resulting from their administration have been in each case erythematous, though in the case of copaiba and belladonna there may be a distinct suggestion of punctation. The general aspect of the case, however, coupled with careful inquiry into the history and the absence of any other signs of scarlet fever, should make its recognition pretty certain.

Diphtheria.- In the diagnosis of diphtheria the fact has been recognised for some years that the presence of exuda. tion or false membrane alone cannot be regarded as the infallible criterion of diphtheria as was taught by Brettonneau in the year 1826 and has been almost universally accepted until quite recently. It is now customary to regard the presence or absence of the Klebs-Löffler bacillus as sufficient to determine the diagnosis. Although in the large majority of cases this relation holds good a more extended observation has shown that the test in actual practice is not by any means so reliable an indication as was originally believed, and this for two reasons. In the first place, not only do we occasionally come across a typical and even severe or fatal attack with distinctive clinical appearances of diphtheria, in which careful and repeated examination of the throat and secretions fails to reveal the presence of a single diphtheria bacillus, but characteristic and even virulent bacilli may not infrequently be found in the throat secretions of perfectly healthy persons, especially if they have been in contact with cases of diphtheria. Indeed, one of the most typical and virulent cultures of diphtheria bacilli which I ever remember to have seen was obtained from the throat of one of my medical colleagues who was at the time and subsequently in his normal health with no suspicion even of a sore-throat. And the same thing may often be demonstrated in respect to the nurses who are working in the diphtheria wards. Now, whatever bearing this may have in regard to their infectivity one would certainly not be justified in saying that these persons were suffering from diphtheria. Again, in a consecutive series of 140 cases of scarlet fever in which the throat secretion was bacteriologically examined diphtheria bacilli were detected by Dr. Sims Woodhead in 36 per cent. of them, although very few presented features which suggested the presence of diphtheria. Ranke of Munich found diphtheria bacilli in 53 per cent. of his scarlet fever cases. Moreover, the more or less persistent rhinorrhœe which is so often seen during convalescence from scarlet fever in young children is in perhaps the majority of cases found to be teeming with bacilli which are morphologically difficult to distinguish from those of true diphtheria. In some of these, at any rate, as was shown by Dr. Todd of the London Fever Hospital, the discharge contained genuine Klebs-Löfler bacilli, as evidenced by their virulence when injected into susceptible animals. It is a curious fact that the bacilli in these cases are almost invariably limited to the nasal discharge and do not invade the faucial or laryngeal mucous surfaces, and though their presence is unattended by any visible exudation or clinical evidence of diphtheria the possibility of their becoming a source of infection under favourable conditions cannot be ignored. Although a negative bacteriological examination cannot unfortunately be regarded as quite conclusive a strong presumption is made out in favour of the case not being one of diphtheria in the hands of an expert observer. The second, and to my mind an even stronger, argument against the infallibility of the bacteriological test is the wide difference of opinion which exists amongst eminent bacteriologists as to what is and what is not a diphtheria bacillus. A bacillus which is regarded by one observer as a true bacillus which has undergone a temporary attenuation is held by another equally experienced observer as a psendobacillus, or one which is of an entirely distinct species and incapable under any circumstances of acquiring virulent pathogenic properties. To the first observer the case is one of diphtheria, potentially at any rate; to the other it is not a case of diphtheria at all. Until the limits of normal variation both in respect to form and virulence can be agreed upon no practical differentiation is possible and as long as an appeal to the results of injection into the lower animals is necessary for a decision the test must remain of limited value in practice.

Having now briefly alluded to the disabilities which attach to the bacteriological test in the diagnosis of diphtheria one may summarise its utility in actual practice as follows. A negative result after careful examination of material taken from the throat and of the culture derived from it on at least two occasions may be held practically to exclude diphtheria. A positive result after examination of a throat presenting a definite pellicular exudation or from the larynx without visible exudation on the fauces may be regarded as sufficient evidence of diphtheria. The presence of short bacilli (suggesting diphtheria bacilli) in the nasal discharge alone even though attended with isolated spots of exudation on the tonsils cannot be held to be distinctive unless confirmed by inoculation into animals.

The procedure required to secure a bacteriological examination is, I have no doubt, familiar to most, and in view of the importance attaching to the early recognition of diphtheria both for successful treatment and for speedy isolation, and having regard to the facility with which the practitioner is now able to get the examination made for him, it is very desirable that he should be prepared with the necessary equipment. All that is required is a couple of testutubes, the one containing a sterilised swab of cotton-wool for taking 
material from the throat and the other some inspissated blood serum, preferably Löffler's preparation, on which the material is sown by simply rubbing its surface with the swab These tubes can be procured at very small cost from the Jenner Institute, the Clinical Research Society, Messrs. Burroughs, Wellcome, and Co., and other druggists of repute, and as they will keep for months in a cool place two or three of them may be kept for immediate use should occasion arise.

In the differential diagnosis of faucial diphtheria it is mainly in connexion with follicular tonsillitis and the exudation throats present in some cases of scarlet fever that difficulty is most of ten experienced. From follicular tonsillitis the differentiation may be most difficult, and, indeed, it may be impossible apart from the bacteriological test. The distinction is rendered none the less difficult owing to the fact that in some cases of diphtheria the exudation commences at the very points which are most characteristic of follicular tonsillitis-viz., at the mouths of the follicular crypts in the form of spots or little patches of exudate which at a later stage enlarge and ultimately become confluent so as to form a continuous pellicle covering the surface of the tonsil, but at an early stage the appearance of both is very similar. The clinical features on which some reliance can be placed are that in follicular tonsillitis the spots rarely coalesce and the exudation never spreads to adjacent parts but remains limited to the tonsils. Both tonsils are usually affected very much to the same degree and the same thing holds good for the swelling of the subjacent glands, while in diphtheria it is perbaps more common for one side to be involved more than the other and for the gland swelling to be proportionally greater. In follicular tonsillitis albuminuria is far less common, but the tempera. ture is usually higher-in fact, the general statement is justified that the constitutional disturbance in follicular tonsillitis is likely to be more pronounced than in a case of diphtheria of sufficient mildness to be mistaken for it. It should be remembered, too, that in a child the attack is more likely, primâa facie, to be one of diphtheria than of tonsillitis simply because in childhood the latter disease is relatively uncommon. Laryngeal implication never super venes, nor does any subsequent paralytic affection, whether of the palate, accommodation, or of the skeletal musclesso distinctive of diphtheria.

Now, of those cases of scarlet fever which are attended with an exudation simulating the appearance of diphtheria it may be stated generally that they are almost invariably severe attacks and the characteristic symptoms of scarlet fever are usually so pronounced that the presence of that disease can hardly be overlooked. The difficulty in this instance is usually to decide whether the exudation is purely scarlatinal or whether, as is sometimes the case, it is an attack of scarlet fever complicated with coexistent diphtheria. The scarlatinal exudation is limited to the tonsils practically always; it is of a cheesy, friable consistence and it is easily removed with a camel-hair brush, tending to disappear spontaneously in a day or two. If it be genuine diphtheria, on the other hand, it is usually thicker, tough, gelatinous, or fibrous-looking, incapable of removal without lacerating the subjacent tissue, and it separates en masse after an interval of several days. In a case of uncomplicated diphtheria, of course, the exudate may be thin, friable, and easily detached, but in these concurrent attacks the condition is usually grave and the exudation as a rule presents the characters which I have mentioned. The constitutional depression, too, is severe and the case is very liable to terminate fatally with cardiac failure or to present some form of paralysis during early convalescence, As far as my experience goes it is much more common for a case of diphtheria attended with an erythematous flush to be mistaken for scarlet fever than for a case of scarlet fever with exudation to be confused with diphtheria.

In laryngeal diphtheria or membranous croup the differential diagnosis from simple laryngeal catarrh may be very difficult during the early stage, and this is particularly so when the larynx is the primary seat of the disease. It is very fallacious to place any reliance on a history of exposure to cold or on the apparent absence of any source of infection. Nor can any trustworthy indication be derived from the character of the respiration or the quality of the cough or laryngeal sound. - I know of no other reliable test than a bacterjological examination of some of the laryngeal mucus which can be easily obtained by passing a swab mounted on a bent wire into the upper part of the larynx. But this, of course, means the loss of valuable time unless the diagnosis can be made by means of an immediate microscopical exa mination of the material on the swab, which is usually possible to anyone conversant with the diphtheria bacillus and which in most cases will prove reliable. The fact that "cases of sinple catarrhal laryogitis are never fatal" is not of much assistance in the early diagnosis of the condition. In cases, however, in which the fauces are involved primarily or even coincidently with the larynx-a condition which is of common occurrence-there is not much room for doubt. The appearance of the very smallest patch of exudation on the tonsil in a case of croup is practically sufficient to establish it as one of laryngeal diphtheria and calls for prompt and $\epsilon$ fficient treatment. This is above all the class of case for showing what a splendid weapon we possess in antitoxin,

Enteric fever.-Let us now turn our attention to enteris fever, a disease of which the diagnosis is liable to be attended with more difficulty probably than in the case of any other specific fever. There are several reasons why this should be so. 1. In its clinical appearances it so often simulates some other disease. 2. There is frequently a complete absence of any symptoms referable to the abdomen consequently a diagnosis can only be arrived at in many cases by a process of exclusion. 3. It is very prone to become complicated by some other disorder, particularly bronchitis, pneumonia, and alcoholism, of which the symptoms are liable to be so prominent as to obscure the ordinary manifestations of enteric fever. 4. Lastly, the indications suggesting the presence of enteric fever may not be apparent until the lapse of a week or more, and, having in the meantime formed a different opinion as to the nature of the attack, we are liable to be bampered in our diagnosis by the influence of a preconceived idea.

In most cases of enteric fever the development of the attack is undoubtedly slow and insidious, but by no means necessarily so. I bave met with a good many cases of enteric fever in which the onset was as sudden and explosive as in any case of small-pox or influenza and these cases almost invariably run a severe course. During the first week of enteric fever, in addition to the usual complaint 0 headache, weariness, aching of the back and limbs, progressive loss of appetite, coating of the tongue, vague pains in the abdomen, and increasing pyrexia, there are four symptoms which are very suggestive of oncoming enteris fever, though not often mentioned, as far as I remember, in the text-books-viz., slight bronchial cough, sore-throat, partial deafness, and bad dreams. A patient will frequently admit upon being interrogated that he has slept badly, often during the night waking suddenly with a start in consequence of some transient nightmare which he bas probably quite forgotten in the morning until it is recalled to his mind by a leading question. On inquiry into the history of the onset it will usually be elicited that there have been short periods of intermittent diarrhcea, rarely of longer duration than a day or two, which may or may not reappear during the progress of the fever. The fact cannot be too strongly emphasised that diarrhoea is by now means a necessary attendant of enteric fever. If 100 con. secutive attacks of all grades of severity be taken it will bo found that, in this country at any rate, the large proportion (if we except the slight premonitory diarrhoea referred to) are characterised by constipation throughout and require the routine soap-and-water enema every third day. If, however. the severe attacks alone be taken the majority will be found to have been attended by diarrhcea of the usual pea-soup character.

of the other characteristic indications of enteric fever a few words must suffice. The typical daily remittent temperature declines gradually during defervescence. A termination by crisis hardly ever occurs in enteric fever unless the case has been complicated, usually by pneumonia. This may affect a small patch of lung simply and not necessarily an entire lobe. It will have been sufficient to delay the normal defervescence of temperature which would otherwise have taken place, but when the pneumonic crisis occurs the temperature falls suddenly, since the enteric fever may no longer be able to provide for its further sustenta tion. A slow pulse is occasionally met with, the rate at no time rising above from 44 to 60 beats, although the temperature may have ranged from $101^{\circ}$ to $103^{\circ} \mathrm{F}$. for a considerable time. These "slow pulse" cases in my experience have always done well, though Murchison seems to have regarded them as of unfarourable omen. The eruption is 
never seen before about the end of the first week, but spots may come out at any time afterwards until the temperature has reached the normal. Their number conveys no indication of the severity of the attack as it does in typhus fever. About 30 per cent. of the cases are unattended by a rash and the large majority of these are in children. One cannot expect to find palpable evidence of enlargement of the spleen until the end of the first week and the enlargement can rarely be detected later than two or three days after the temperature has begun to fall. Personally, I am very sceptical as to my ability to recognise enlargement of the spleen in enteric fever with certainty unless I can feel its lower border beneath the costal arch. I have often seen the observation as to the absence of enlargement discounted in the post-mortem room. Gbvious desquamation occurs in from 30 to 40 per cent. of enteric fever convalescents. It is for the most part limited to the trunk, is scaly, and is never, I believe, of the "pindrole" variety.

During recent years a great deal of interest has centred cound what is usually known as Widal's reaction (it should more justly be called the Gruber-Durham reaction, though Widal was the first to apply it as a test for the recognition of enteric fever). It may be briefly described as the faculty which is possessed by the blood serum of a person suffering from, or recently convalescent from, enteric cever of causing the bacilli in a 24 hours' broth culture to lose their motility and range themselves together into clumps, instead of remaining in active movement as isolated individuals spread all over the field. Now, the possession of this power of causing agglutination by enteric serum may with certain restrictions be regarded as a test of distinct value. It must be remembered that normal blood serum undiluted, or if but slightly diluted, will after a time produce a certain degree of clumping, and that the bacilli in a drop of culture when placed on the slide will after a time lose their motility and often evince a tendency to clump without any admixture of enteric serum at all. Hence a standard degree of dilution for the serum and a standard Eength of time within which the reaction must occur have to be fixed before any conclusion can be drawn as to the claims of any particular serum. A dilution of one in 10 and a period of half an hour have until recently been the standards adopted, though a dilution of one in 20 or one in 30 is now cegarded as more reliable.

In order to illustrate the degree of reliance which may be placed on the serum test I cannot do better than summarise the results obtained in two series of cases which were rested by separate observers. The first, consisting of 125 eases was published by Dr. Colville and Dr. Donnan of Belfast in the British Medical Journal in 1898, and the second, comprising 184 cases, was reported by Dr. Kirton of the Brook Hospital in the Medical Supplement to the Metropolitan Asylums Board Report for 1897. Of the total 309 cases examined, 206 were proved to have been enteric fever either by undoubted clinical or post-mortem evidence and 103 were decided not to be enteric fever either at the vime or as the result of more extended clinical observation. of the 206 cases of enteric fever the blood serum gave the reaction in 201 (although it is fair to state that in seven of them the reaction was not very complete), and of the 103 cases which were not enteric fever in only one did the reaction occur and that, curiously enough, was a case of typhus fover-that is to say, in only six out of 309 cases, or rather less than 2 per cent., did the serum test fail to support the diagnosis. The reaction, it should be remembered, is rarely obtainable before the sixth or seventh day of the attack and in some cases the serum ceases to react before the expiration of three months from its commencement, though it can of ten be obtained for two years or more after recovery, but usually in a less complete degree. This fact should lead to careful inquiry into the previous history. Though by no means difficult of application there are probably few practitioners who are likely to keep by them the necessary equip. ment, which includes a living culture of enteric fever bacilli of not more than 24 hours' existence. In view of the ease, however, with which one can get the examination made at the Jenner Institute or at other accredited laboratories it is well to keep at hand the means necessary for collecting and transmitting a few drops of the blood of any suspected case. All that is required is a sterilised capillary pipette (Pakes's tube), a little ether, and a sharp needle.

Now, of the diseases which are most likely to be mistaken for enteric fever and, indeed, frequently are mistaken for it, perhaps the most important are, first, a group of affections characterised by abdominal inflammation-viz. febrile gastro-intestinal catarrh (particularly in young children), tuberculous peritonitis, tuberculous enteritis, ulcerative colitis (which may have been set up by an attack of enteric fever), and appendicitis; and, secondly, certain general diseases, especially acute miliary tuberculosis, meningitis (most of ten tuberculous), pneumonia, ulcerative endocarditis typhus fever, acute alcoholism, and influenza. All these, and a good many others, I have seen mistaken for enteric fever, and often with very good reason, at an early stage. Tempting though it is to enter into a consideration of their detailed specific distinctions, I fear that the time at my command is far too restricted to admit of it. I must therefore content myself with recalling to mind certain positive indications which point to the diagnosis of enteric fever and which in combination must be rare accompaniments of any of the affections which I have enumerated. 1. Some degree of deafness unattended with evidence of mental impairment. 2. Continued pyrexia of a regular daily remittent character. rarely showing any period of complete intermission until defervescence which sets in gradually. 3. A papular rash on the trunk, rarely affecting the limbs and still more rarely the face. 4. Enlargement of the spleen to an extent often palpable to the fingers. 5. A positive reaction with the Widal test. 6. The onset of intestinal hæmorrhage or perforation at a later stage.

The reappearance of epidemic influenza during the last 10 years has greatly increased our difficulties. There are some attacks of that now "hardy annual" which I will defy any human observer to differentiate from enteric fever at an early stage; that is unless he may have seen very little of either. The differentiation from typhus fever is not of the same importance in this country as it was 25 years ago but when the disease occurs in London, as it occasionally does even now, the earlier cases are usually mistaken for enteric fever, especially in children, in whom the rash is often absent.

Small-pox. - The diagnosis of small-pox at an early stage is liable to be confused with measles and also with scarlet fever when attended with a prodromal rash of an erythematous or punctiform character. In the former case the similarity may be very striking, especially if the onset of measles be attended with lumbar pain as is sometimes the case in adults. The absence of vomiting, however, and of the peculiar shotty feel which the rash of small-pox soon assumes and the fact that the temperature rises instead of falls when the eruption comes out and that it is accompanied by marked catarrhal symptoms will soon dispel any doubts which may remain. But it is in connexion with chicken-pox that mistakes are most often made in the diagnosis of smallpox, especially in the modified form of the disease.

The points of difference in connexion with the development of the attack and the distribution and characters of the respective eruptions are well set out in the text-books and are adequate for a diagnosis in most cases, but the fact that chicken-pox in an adult is apt to be attended with really severe symptoms of invasion and that in modified small-pox the specific distinctions in respect to the eruption may be by no means easy of recognition still leaves room for a very considerable degree of doubt in some cases. There are, bowever, three points which rarely seem to be insisted on even if they receive any mention at all in the text-books. They are well worthy of remembrance as any one of them may be in itself almost sufficient to clinch the diagnosis. 1. In varicella, pocks in different stages of developmentpapule, vesicle, pustule, and scab-may usually be seen at the same time within a circumscribed area of skin. 2. If a single developed vesicle can be detected upon any point of the skin which 24 hours previously showed no sign of a developing pock even in its early papular stage, that one vesicle is sufficient to exclude small-pox altogether. 3 . The detection of any cicatrices of antecedent varicella (which on careful scrutiny of the surface are to be found much more frequently than is usually credited) is practically sufficient to exclude varicella, so extremely rare is a second attack of that disorder.

Stockwell, S.W

SdPeranndation Allowance.-Dr. Richard Purnell, late medical officer of the First District Wells (Somerset) Union, has been granted a superannuation allowance of about $£ 70$ by the Wells Board of Guardians. 


\section{PHYSICAL DIAGNOSIS IN THE INSANE.}

BY JAMES F. GEMMEL, M.B., C.M. GLASG.

ASSISTANT MIEDICAT OFTICER TO THE CUUNTY ASTLUN, IANCASTER.

NOWHERE perhaps is the importance of physical diagnosis more forcibly presented to our minds than in the insane. In them we have much more frequently than not it alone to depend upon, and the aid which the general practitioner and hospital physician obtain subjectively, together with the history, is usually wanting or at best unreliable. This of itself is a great disadvantage and we would be grateful were it the only one, for another which the asylum physician has frequently to encounter is the resistance of the patient to examination. Only those who have experienced it know what this really means. The maniacal shouts, turnings, and twistings, to say nothing of more determined efforts to oppose, are circumstances not calculated to facilitate examination. Yet such we have frequently to contend against, not only in diagnosing disease in this class of patient but in treating it. Catheterism, for example, has often to be performed and wounds dressed in patients assuming the most inconvenient postures. Another drawback, the insensitiveness of the insane, from whatever cause arising, to bodily pain and discomfort, is sufficiently well known. The numerous instances of retention of urine suspected by the practised eye from perhaps a change in posture or an alteration in walk; strangulated hernia uncomplained of and discovered on bathing or on attention being called to an attack of vomiting; enteric fever recognised when the eruption is out; and numerous other examples of serious bodily disease to which no attention has ever been directed by the patient himself, are experiences which we have all met with. Hence it is that apparently very trivial circumstances may often give warning and indicate to our eyes grave illness. Notwithstanding, however, the keenest observation we have every now and then disease presented to us in a stage which in sane patients would have been complained of at the outset and have thus afforded an earlier opportunity of diagnosis or at all events an approximate one. For the detection, then, of disease in the insane we have to rely almost entirely upon physical diagnosis. With these preliminary remarks $I$ propose to mention and briefly describe a few cases by way of illustration, some of them interesting from their comparative rarity and all with one exception from the fact that the history and symptomatology were for practical purposes wanting.

Purulent meningitis. - The patient in this case was a woman, aged 21 years. Beyond the fact that she was known to have a mitral regurgitant murmur there was nothing in her past history having any bearing on her present ailment, of which the patient was quite incapable of giving any account. She had for a few days previously to Sept. $28 \mathrm{th}, 1898$, not been taking her food as well as usual and as she looked generally ill on that morning she was at once sent to the hospital. Her temperature then was $103^{\circ} \mathrm{F}$. She gave no indication that she could hear what was said to her, was semi-conscious, and could scarcely be roused. Her muscular system was in a state of extreme irritability and on being raised for purposes of examination she became very stiff and rigid, the head was drawn back, and the muscles of the back of the neck were thrown into a state of tetanic contraction. The pupils were dilated and reacted sluggishly, the gums were spongy and suppurated from decayed teeth, the tongue was dry and coated, and the urine was normal. Nothing was discoverable in the lungs or abdominal organs to account for her condition. Her temperature in the evening rose to $105^{\circ}$ and since admission frequent retching and romiting had supervened. On the 30th the temperature still continued high $\left(104^{\circ}\right)$; vomiting, irrespectively of the state of the stomach as to food, was persistent; the bowels were confined; there was retention of urine; the pupils were still dilated; the tongue was dry and foul and the lips and gums were covered with sordes. The muscular system was extremely irritable and the patient became rigid on any attempt at movement. The pulse was rapid but regular and the respirations were correspondingly increased. There was a dark trophic patch on each buttock. The patient was quite unconscious. On Oct. 1st the temperature was $104.8^{\circ}$; the specific gravity of the uxine was 1030 , it was albuminous, and contained a trace of sugar; the head and eyes deviated to the right, the mouth was slightly twisted to the same side, and there was slight facial twitching. The right arm was rigidly flexed and when forcibly extended and let go at once resumed its abnormal position. The eyes were suffused. There were dark trophic areas and bullæe on the heels and tarsal prominences. On the 2nd the patient was comatose and rapidly sinking. She died on the evening of the $3 \mathrm{rd}$, the temperature rising to $105.8^{\circ}$ some hours before death.

Neoropsy.-The dura mater was thickened and firmly adherent to the skull cap. The pia arachnoid over the convex and basal aspects of the brain was thickened and infiltrated with a greenish-yellow exudation of pus dipping down between the sulci and specially thick and abundant in the track of the larger fissures. The surface and lateral margins of the cerebellum were also invaded. Generally the brain substance was soft and cedematous anc the vessels were congested. The grey matter was of a deeper colour than usual. The ventricles were dilated and filled with a dirty yellow fluid. There were hypertrophy of the left ventricle and thickening of the mitral valve. The kidneys were congested and the left one exhibited several recent infarcts.

Phlegmonous gastritis.-A man, aged 66 years, or Dec. 22nd, 1896, complained of nausea and vomiting. There was nothing, however, in his general appearance to indicate that there was anything very much the matter. His temperature was normal but the pulse was somewhat irregular. The next morning he rose but fell back and suddenly expired.

Necropsy.-The heart was found to be enlarged, weighing 14 ounces; the mitral valve was stenosed and the aortic valve was calcareous and incompetent. The stomach was greatly dilated, the mucous membrane was congested, and submucous hæmorrhages were present. The stomach wall was greatly thickened and on section large quantities of fluid pus exuded from the submucosa which was in a state of diffuse purulent infiltration.

Ifalignant endocarditis verrucosa.-No detailed description of this case which lasted about three weeks will be entered upon, but only a general survey of its progress from com. mencement to finish along with the factors contributing to its differential diagnosis. The only clear history obtainable was that some months previously the patient had a severe attack of rheumatic fever from which she slowly recovered, but with an impaired mental condition which gradually becoming more pronounced culminated before admission in an attack of acute delirium. Her condition on admission was: one of semi-consciousness, extreme restlessness, incoherent. muttering and screaming, loss of control over the sphincters, constant purposeless movements of the limbs and body, and extreme motor irritability. In fact, at first sight the case appeared to be one of severe and fatal chorea which is occasionally to be met with. That there were some grounds for the suspicion I think may not very strongly be objected to. The patient sometimes refused food, but this was not owing to inability to swallow, as the act of deglutition was normally performed even when the general muscular system was in a state of violent movement, the patient tossing her arms and legs about and rolling her head uneasily from side to side. These choreiform movements would suddenly cease and the patient drop into apparently a deep slumber from which, however, she was easily roused. The skin was hot and dry and later exhibited a petechial rash; the tongue and lips were parched and covered with sordes; the pupils were dilated and her general appearance indicated some acute febrile affection. The temperature was $103^{\circ} \mathrm{F}$. on the evening of admission and continued more or less elevated throughout, though exhibiting considerable irregularity. The urine contained a large quantity of albumin and there was a loud mitral regurgitant murmur considerable diarrhœea, slight distension of the abdomen, and gurgling were also present from the beginning. No rose spots or enlargement of the spleen were ever detected. Towards the end the heart's action became extremely rapid; the albumin increased, and coma supervened. Throughout muscular irritability was extreme and any attempt at sitting the patient up was accompanied by tetanic rigidity of the erector spina muscles. Passive motion of the extremities yielded the same state of matters.

Necropsy.-The heart weighed eight ounces. Large warty vegetations were present on the mitral segments and surrounding the mitral orifice. The left kidney and spleen were the seat of infarctions and both the kidneys were cirrhotic. 